\title{
Novel Selection Criteria of Primary Side Transistors for LLC Resonant Converters
}

\author{
Maria R.Rogina \\ Electronic Power Supply Systems Group \\ University of Oviedo \\ Campus de Viesques, 33204. Gijon, Spain \\ rodriguezrmaria@uniovi.es
}

\author{
Alberto Rodriguez \\ Electronic Power Supply Systems Group \\ University of Oviedo \\ Campus de Viesques, 33204. Gijon, Spain \\ rodriguezalberto@uniovi.es
}

Piet Vanmeerbeek

Power Technology Centre, Corporate R\&D

ON Semiconductor

Oudenaarde, Belgium

piet.vanmeerbeek@onsemi.com

\author{
Diego G.Lamar \\ Electronic Power Supply Systems Group \\ University of Oviedo \\ Campus de Viesques, 33204. Gijon, Spain \\ gonzalezdiego@uniovi.es
}

Filip Bauwens

Power Technology Centre, Corporate $R \& D$

ON Semiconductor

Oudenaarde, Belgium

filip.bauwens@onsemi.com

\begin{abstract}
An insightful evaluation of commercial highvoltage power MOSFETs for soft-switching converters is reported in this paper with special emphasis on elucidating power loss contributions and electrical parameter requirements. Experimental tests have been carried out to evaluate different Silicon Super-Junction technologies used in the primary side of a $600 \mathrm{~W}$ half-bridge $L L C$ resonant converter operating in inductive mode. Unexpectedly, none of the existing figures of merit, inferred from datasheets, can predict the performance ranking due to the additional soft-switching losses (Esw_soft). Subsequently, a new characterization test based on a pulsed I-V system is suggested and proved to quantify Esw_sofT.
\end{abstract}

Keywords-LLC, figure of merit, soft-switching, superjunction MOSFET

\section{INTRODUCTION}

Resonant circuits have a long history in power conversion [1]. However, it was not until last decade that the market adoption of LLC converters (Figure 1) was massive in the segments of adapters, flat panel TV, datacenters, Electric and Hybrid Vehicle (EV/HEV) and Photovoltaic (PV) inverters among others [2], [3]. Many of these applications must meet the highest efficiency standard, such as, 80PLUS® Titanium [4]. Consequently, the efficiency of the selected converter topology must be very high and the selection of a resonant topology achieving Zero Voltage Switching (ZVS) can provide a reduction of losses and volume. Nowadays, the proper selection of high-voltage $(\sim 600 \mathrm{~V})$ Super Junction MOSFETs (SJ-MOSFETs) used in the LLC primary side is crucial to obtain an optimum system design with a small footprint, low cost and high efficiency [5].

This work aims to review the methodology followed to select SJ-MOSFETs by deeply investigating their different power losses contributions. This means interpreting all the power loss contributions and which are the electrical parameters that have direct impact in them. Besides, new characterization techniques and Figures of Merit (FoMs), that will help the designer during the selection of the devices for the application, are proposed.

This paper is organized as follows. Section II explains the criterion to select the SJ-MOSFETs under comparison. Moreover, a detailed insight on the power losses contributions of the SJ-MOSFETs is carried out (losses models are developed) in order to compare them with the obtained experimental results. In Section III a new electrical characterization is proposed and a new FoM which takes into account soft-switching losses is proposed. Finally, conclusions are drawn in Section IV.
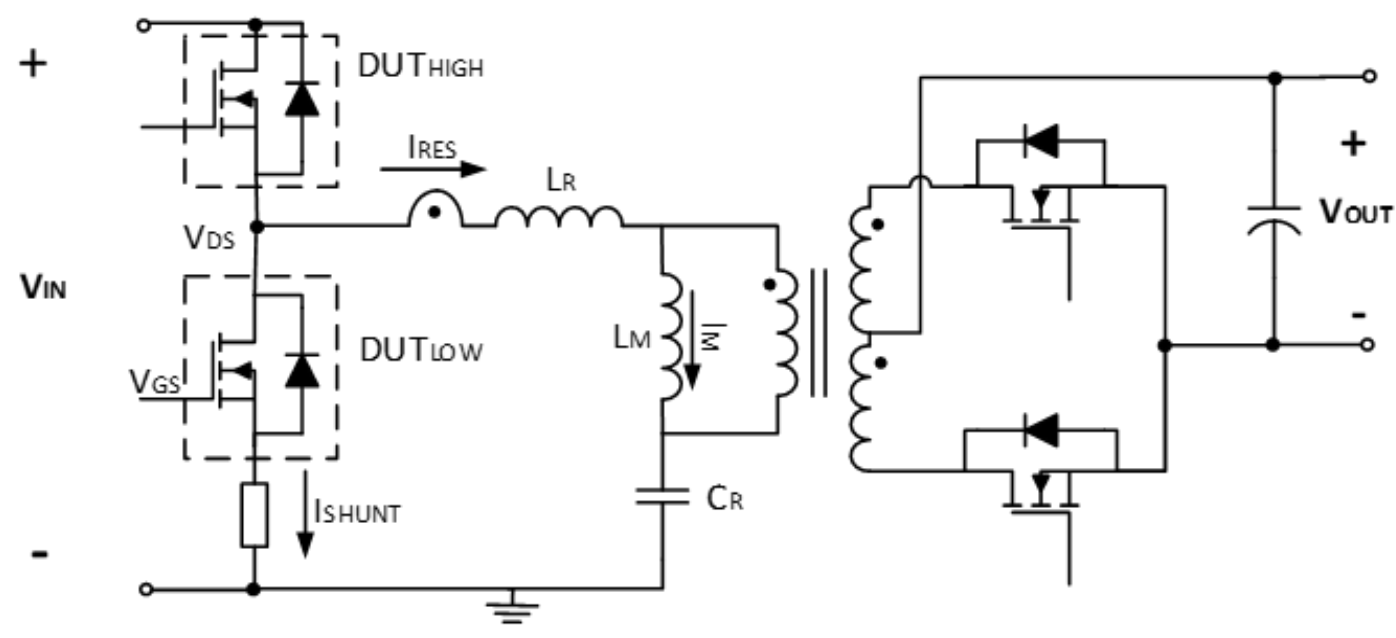

Figure 1 Simplified circuit scheme for LLC resonant converter and sensing method 
Table 1 List of SJ-MOSFETs explicitly for LLC primary side with main electrical characteristics and FoMs. All devices are packaged in TO-220 except DUT4 in TO-220FP

\begin{tabular}{|c|c|c|c|c|c|c|c|c|c|c|c|c|c|}
\hline $\begin{array}{c}\text { DUT } \\
\text { (SJ_MOSFET) }\end{array}$ & $\begin{array}{c}\mathbf{R}_{\mathrm{ON}} \\
(\mathrm{m} \Omega)\end{array}$ & $\begin{array}{c}\mathbf{B V}_{\text {DSS }} \\
\text { (V) }\end{array}$ & $\begin{array}{l}\mathbf{V}_{\mathrm{TH}} \\
(\mathbf{V})\end{array}$ & $\begin{array}{l}\mathbf{R}_{\mathrm{G}} \\
(\mathbf{\Omega})\end{array}$ & $\begin{array}{c}\mathbf{Q}_{\mathrm{G}} \\
(\mathrm{nC})\end{array}$ & $\begin{array}{l}\mathbf{Q}_{\mathrm{GD}} \\
(\mathrm{nC})\end{array}$ & $\begin{array}{r}\mathbf{Q}_{\mathrm{GS}} \\
(\mathrm{nC})\end{array}$ & $\begin{array}{l}\mathbf{E}_{\text {OSs }} \\
(\mu \mathrm{J})\end{array}$ & $\begin{array}{l}\text { Qoss } \\
\text { (nC) }\end{array}$ & $\begin{array}{l}\mathbf{R}_{\mathrm{ON}} * \mathbf{Q}_{\mathrm{G}} \\
\left(\Omega^{*} \mathrm{nC}\right)\end{array}$ & $\begin{array}{c}\mathbf{R}_{\mathrm{ON}} * \mathbf{Q}_{\mathrm{GD}} \\
\left(\Omega^{*} \mathrm{nC}\right)\end{array}$ & $\begin{array}{c}\mathbf{R}_{\mathrm{ON}}^{*} \mathbf{E}_{\mathrm{OSS}} \\
\left(\mathbf{\Omega}^{*} \boldsymbol{\mu} \mathbf{J}\right)\end{array}$ & $\begin{array}{c}\mathbf{R}_{\mathrm{ON}} * \mathbf{Q}_{\mathrm{Oss}} \\
\left(\mathbf{\Omega}^{*} \mathrm{nC}\right)\end{array}$ \\
\hline 1 & 155 & 600 & 3.5 & 0.9 & 24 & 8 & 5 & 2.7 & 140 & 3.7 & 1.2 & 0.4 & 21.7 \\
\hline 2 & 168 & 650 & 4 & 0.6 & 60 & 25 & 12 & $\begin{array}{l}6.4 \\
\end{array}$ & 121 & 10.1 & 4.2 & 1.1 & 20.3 \\
\hline 3 & 171 & 600 & 4 & 3.4 & 37 & 13 & 11 & 4.9 & 106 & 6.3 & 2.2 & 0.8 & \begin{tabular}{ll|}
18.1 \\
\end{tabular} \\
\hline 4 & 160 & 650 & 3.5 & 6 & 31 & 10 & 12 & 3.6 & 124 & 5.0 & 1.6 & 0.6 & $\begin{array}{ll}19.8 \\
\end{array}$ \\
\hline 5 & 168 & 600 & 3 & 7 & 29 & 12 & 6 & 4.1 & 123.8 & 4.9 & 2.0 & 0.7 & 20.8 \\
\hline 6 & 175 & 600 & 3 & 7 & 29 & 12 & 6 & 4.6 & 122.4 & 5.1 & 2.1 & $\begin{array}{l}0.8 \\
\end{array}$ & 21.4 \\
\hline
\end{tabular}

\section{APPLICATION MEASUREMENTS AND BENCHMARKING}

A group of SJ-MOSFETs (with similar values of conduction resistance, $\mathrm{R}_{\mathrm{ON}}$, and output charge, Qoss) from different manufacturers (Table 1) was tested in a commercial evaluation board featuring a $600 \mathrm{~W} / 200 \mathrm{kHz}$ half-bridge LLC resonant converter with input voltages $\left(\mathrm{V}_{\mathrm{IN}}\right)$ between $350-410$ $\mathrm{V}_{\text {DC }}$ and a fixed output voltage ( $\mathrm{V}_{\text {OUT }}$ ) of $12 \mathrm{~V}_{\text {DC. }}$ [6]. All the SJ-MOSFETs under examination are recommended for LLC resonant converters by the major vendors. The basic requirements of the SJ-MOSFETs related to a fixed resonant tank $\left(\mathrm{L}_{\mathrm{M}}, \mathrm{L}_{\mathrm{R}}, \mathrm{C}_{\mathrm{R}}\right)$ and deadtime $\left(\mathrm{t}_{\text {dead }}\right)$ are fulfilled by all transistors in order to ensure ZVS inductive mode test during the entire load range.

A minimum deadtime between the turn-off of one primary-side SJ-MOSFET and the turn-on of the other one must be assured to avoid short-circuits (time domain analysis (1), (2)).

$$
\begin{array}{r}
t_{d, \min }=\frac{t_{l i n}}{2}+\frac{2 \cdot Q_{O S S}}{I_{M p k}} \\
t_{l i n}=f\left(R_{G}, V_{G S t h}, \frac{C_{D S}}{C_{G D}}\right)
\end{array}
$$

$\mathrm{t}_{\mathrm{d} \text {,min }}$ is the minimum deadtime needed, proportional to $\mathrm{t}_{\text {lin }}$ (the linear time of operation of the SJ-MOSFETs), Qoss (output charge of the SJ-MOSFETs) and $\mathrm{I}_{\mathrm{Mpk}}$ (peak magnetizing current of the transformer). $t_{\text {lin }}$ is function of $R_{G}$ (gate resistance), $\mathrm{V}_{\mathrm{GSth}}$ (gate threshold voltage), $\mathrm{C}_{\mathrm{DS}}$ (drainto-source capacitance) and $\mathrm{C}_{\mathrm{GD}}$ (gate-to-drain capacitance).

Moreover, the energy in the resonant tank must be enough to discharge the output capacitance of the SJ-MOSFET (energy domain analysis (3)).

$$
\left.\begin{array}{c}
E_{\text {res,min }}=\frac{1}{2}\left(L_{M}+L_{R}\right) I_{M \min }^{2} \\
E_{\text {cap } \text { max }}=\frac{1}{2}\left(2 C_{O S S}\right) V_{D S \max }^{2} \\
>E_{\text {cap } \text { max }}
\end{array}\right\} E_{r e s, \text { min }}
$$

$E_{\text {res,min }}$ is the minimum energy required in the resonant tank, which is proportional to $L_{M}$ (magnetizing inductance of the transformer), $L_{R}$ (leakage inductance of the transformer) and $I_{M \min }$ (minimum magnetizing current of the transformer). The maximum energy that the SJ-MOSFET must store $\left(E_{\text {cap,max }}\right)$ depends on $C_{O S S}$ (its output capacitance) and $V_{D S \max }$ (the maximum drain-to-source voltage in the switching node).

It is not necessary to design a different $L_{M}$ for each SJMOSFET in order to optimize the performance since the devices that have been selected share the same $R_{O N}$ and $Q_{O S S}$ values, and ZVS is guaranteed for all the power range.

Examples of experimental waveforms measured in the converter are shown ( $\mathrm{I}_{\mathrm{SHUNT}}, \mathrm{V}_{\mathrm{GS}}, \mathrm{V}_{\mathrm{DS}}$ and estimated $\mathrm{P}_{\text {ins }}$ in Figure $2 \mathrm{a}$ and $\mathrm{I}_{\text {RES }}$ in Figure $2 \mathrm{~b}$ ) for different load levels. It is worth to remark that even if ZVS is achieved $\left(t_{d, m i n}\right.$ is assured and there is no ringing in the measurements) some power losses appear during the switching (see $\mathrm{P}_{\text {ins }}$ in Figure 2a). Moreover, the IRES value during the transition is nearly the same regardless of the load level, which will be helpful in order to estimate the switching losses.

An efficiency comparison is performed in the full load range with the same test protocol and operating conditions $\left(\mathrm{V}_{\text {IN }}=380 \mathrm{~V}, \quad \mathrm{~V}_{\text {OUT }}=12 \mathrm{~V}\right)$. In Figure 3a, differential efficiencies for loads going from $20 \%$ to $100 \%$ of full load are presented). DUT1 is considered as the reference, since it shows the best performance in the whole range. The dispersion error is estimated considering the information given by the LLC evaluation board manufacturer and the lab equipment utilized.

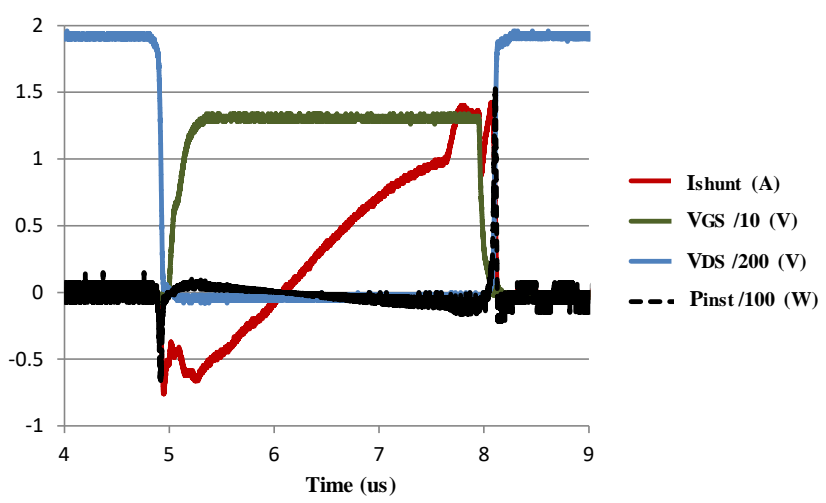

(a)

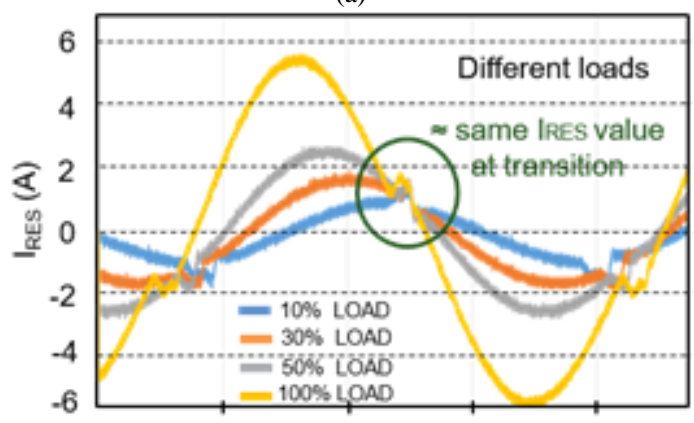

Time ( $\mu s)$

(b) 
Figure 2 (a) Measured waveforms: $\mathrm{I}_{\mathrm{SHUNT}}(\mathrm{A})$ in red, $\mathrm{V}_{\mathrm{GS}} / 10$ (V) in green, $\mathrm{V}_{\mathrm{DS}} / 200(\mathrm{~V})$ in blue and calculated $\mathrm{P}_{\text {inst }} / 100$ (W) in black. (b) Experimental $\mathrm{I}_{\mathrm{RES}}$ measured at different loads.
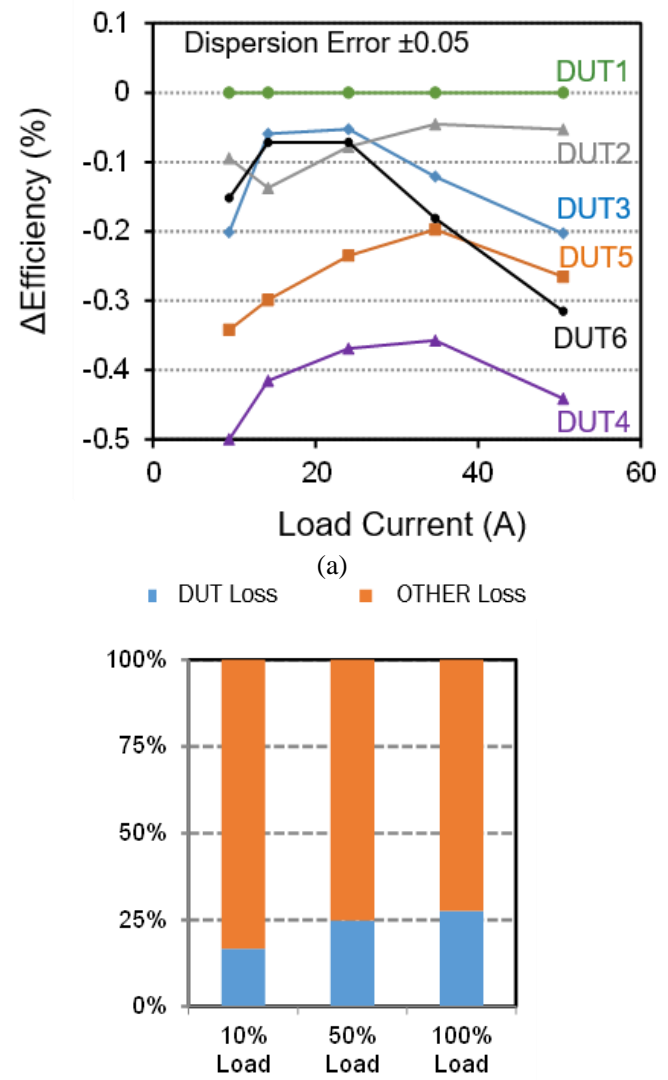

(b)

Figure 3 (a)Measured variation of efficiency with respect to the best SJMOSFET. (b) Measured system power loss contribution from the two primary-side transistors (DUT3)

Figure $3 \mathrm{~b}$ shows that primary-side SJ-MOSFETs dissipate $20-30 \%$ of the system total loss based on the experimental measurements obtained, while the other 70$80 \%$ of the losses are spread out among the magnetics, layout, secondary-side devices, digital circuitry, etc. This makes imperative a proper selection of the primary-side devices.

The extraction of experimental $\mathrm{I}_{\mathrm{SHUNT}}, \mathrm{V}_{\mathrm{DS}}, \mathrm{V}_{\mathrm{GS}}$ and $\mathrm{I}_{\mathrm{G}}$ waveforms (Figure 1) allows to estimate contributions from switching $\left(\mathrm{P}_{\mathrm{SW}}\right)$, driving $\left(\mathrm{P}_{\mathrm{DR}}\right)$ and conduction $\left(\mathrm{P}_{\mathrm{ON}}\right)$ power losses of those primary-side SJ-MOSFETs (Figure 4) under different power load requirements $(60 \mathrm{~W}, 300 \mathrm{~W}$ and $600 \mathrm{~W}$ Figure $4 a, b, c$, respectively).

Even performing ZVS, $\mathrm{P}_{\mathrm{SW}}$ losses are relevant and differences in the power losses between transistors are due to $\mathrm{P}_{\mathrm{SW}}+\mathrm{P}_{\mathrm{DR}}$ at light loads (Figure $4 \mathrm{a}$ ) and to $\mathrm{P}_{\mathrm{SW}}+\mathrm{P}_{\mathrm{ON}}$ at heavy loads (Figure 4c). Traditional selective procedures for power devices, based on only one FoM, for example, the conduction resistance $\left(\mathrm{R}_{\mathrm{ON}}\right)$ are not sufficient since they cannot explain differences among efficiencies for low and medium loads (Figure 3a).

In Figure $4 a$, at low load, whereas low $\mathrm{P}_{\mathrm{ON}}$ losses remain almost equal for all the DUTs, differences in $\mathrm{P}_{\mathrm{DR}}$ losses have small impact and $\mathrm{P}_{\mathrm{SW}}$ losses are dominant.

Besides, at medium load (Figure 4b), divergence in this parameter $\left(\mathrm{P}_{\mathrm{Sw}}\right)$ among devices make the difference $\left(\mathrm{P}_{\mathrm{ON}}\right.$ losses are the highest but fairly the same value, but differences at $\mathrm{P}_{\mathrm{SW}}$ have a great impact in the losses contribution). However, it is known that $\mathrm{P}_{\mathrm{Sw}}$ does not depend only on one electrical parameter ( $E_{\text {oss }}, Q_{\text {oss }}$, etc... in Table 1) and, therefore, new FoMs are mandatory in order to evaluate divergence of power losses.

For heavy loads (Figure 4c), $\mathrm{P}_{\mathrm{ON}}$ is by far the main factor of losses in the SJ-MOSFETs, yet disparity among the $\mathrm{P}_{\mathrm{SW}}$ losses are discernible.

Therefore, depending on the application and the range of power that will be demanded a suitable selection criterion based on more complex FoMs is needed.

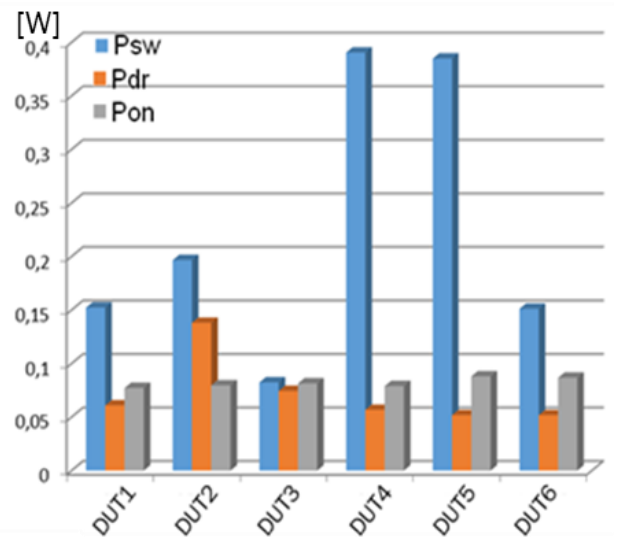

(a)

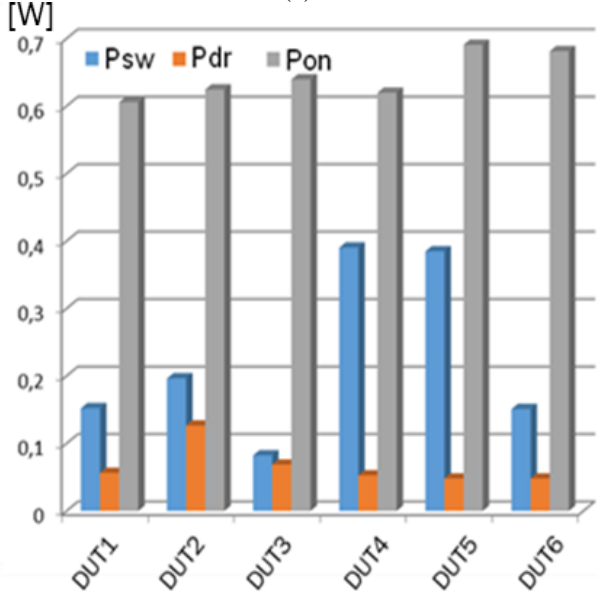

(b)

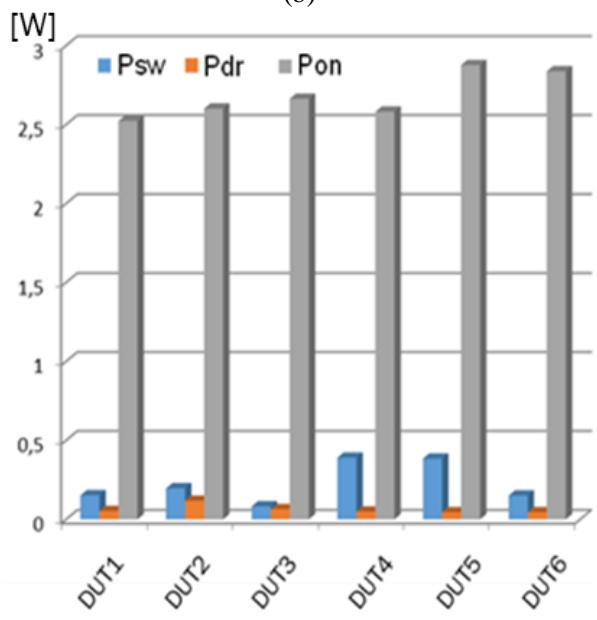

(c)

Figure 4 Measured power loss due to driving $\left(\mathrm{P}_{\mathrm{DR}}\right)$, switching $\left(\mathrm{P}_{\mathrm{SW}}\right)$ and conduction $\left(\mathrm{P}_{\mathrm{ON}}\right)$ at (a) $10 \%$, (b) $50 \%$ and (c) $100 \%$ load per primary-side SJ-MOSFET 
All power losses contributions have been measured experimentally and some losses models have been developed. $\mathrm{P}_{\mathrm{SW}}$ losses are calculated by means of the energy dissipated during turn-on and turn-off transitions (4). It is almost constant for different load levels, since there is little dispersion in $\mathrm{I}_{\text {RES }}$ (Figure 1) value during transitions (Figure 2b).

$$
\begin{aligned}
& E_{S W}=E_{O N}+E_{O F F}= \\
& \int_{<t u r n} I_{S H U N T} \cdot V_{D S} \cdot d t+\int_{\substack{<t u r n \\
\text { off }>}} I_{S H U N T} \cdot V_{D S} \cdot d t
\end{aligned}
$$

Little dispersion $(<5 \%)$ between $\mathrm{P}_{\mathrm{ON}}$ experimental and theoretical values is achieved (Figure 5a), based on $\mathrm{R}_{\mathrm{ON}}$ and ISHUNT waveforms, guaranteeing a fitting model of losses.

However, there are many factors to consider when calculating $\mathrm{P}_{\mathrm{DR}}$ (11) [7] (Figure 5b,c), such as: $P_{d 1}(5), P_{d 2}(6), P_{d 3}(7), P_{d 4}(8)$ (losses due to the commercial driver IC itself, commonly disregarded), driving-on (9) and driving-off losses (10).

The factors that contribute to the total driving power dissipation are:

- The quiescent current (high side and low side) of the IC.

$$
\begin{aligned}
& P_{d 1}=I_{D D \max } \cdot V_{D D \max } \\
& P_{d 2}=I_{Q B S} \cdot V_{B S \max }
\end{aligned}
$$

where $\mathrm{I}_{\mathrm{DDmax}}$ is the operating current for the maximum switching frequency of the application, $\mathrm{V}_{\mathrm{DDmax}}$ the maximum supply voltage of the driver, $\mathrm{I}_{\mathrm{QBS}}$ the quiescent current of the high side section and $\mathrm{V}_{\mathrm{BSmax}}$ the maximum voltage at the bootstrap capacitor.

- The input sections that generate losses by means of their input structures (pull-down resistors, $\mathrm{R}_{\text {pull-down }}$ ).

$$
P_{d 3}=\frac{V_{I N d r i v e r}^{2}}{R_{\text {pull-down }}}
$$

- The leakage losses between the control to any high side section.

$$
P_{d 4}=I_{L V S} \cdot V_{I N}
$$

where $\mathrm{I}_{\mathrm{LVS}}$ is the leakage current and $\mathrm{V}_{\mathrm{IN}}$ is the high side DC voltage.

- The output sections, driving-on $\left(\mathrm{P}_{\mathrm{on}}\right)$ and driving-off $\left(\mathrm{P}_{\text {off }}\right)$ losses:

$$
\begin{aligned}
& P_{\text {On }}=Q_{G} \cdot V_{D D} \cdot f_{s} \cdot \frac{R_{\text {Gon }}}{R_{\text {Gon }}+R_{G}+R_{\text {Gint }}} \\
& P_{\text {Off }}=Q_{G} \cdot V_{D D} \cdot f_{s} \cdot \frac{R_{\text {Goff }}}{R_{\text {Goff }}+R_{G}+R_{\text {Gint }}}
\end{aligned}
$$

where $\mathrm{Q}_{\mathrm{G}}$ is the total gate charge of the power transistor, $\mathrm{f}_{\mathrm{s}}$ the switching frequency and $\mathrm{R}_{\mathrm{Gon}}, \mathrm{R}_{\mathrm{G}}, \mathrm{R}_{\mathrm{Gint}}$ and $\mathrm{R}_{\mathrm{Goff}}$ are the gate and MOSFET resistances detailed in Figure 6.

All contributions can be estimated as the sum of the above-mentioned factors. The final power dissipation is:

$$
P_{D R}=P_{d 1}+P_{d 2}+P_{d 3}+P_{d 4}
$$

As an example of this driving power losses model, a comparison between theoretical and experimental driving-on losses calculation is shown (Figure 5c).

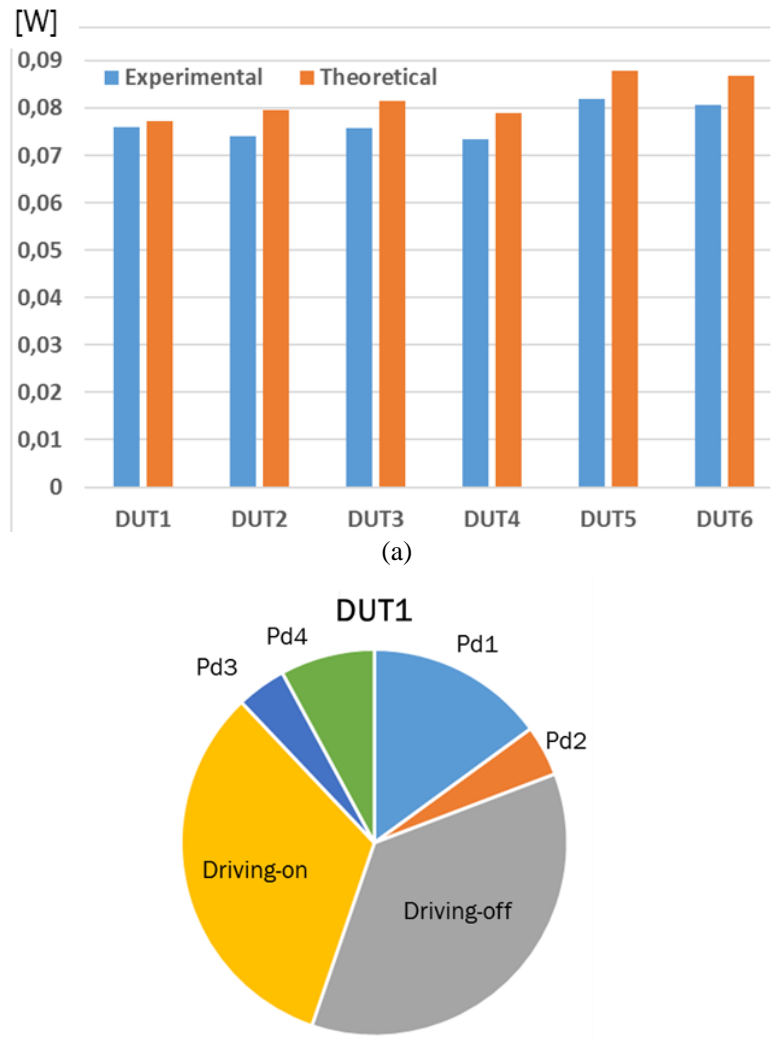

(b)

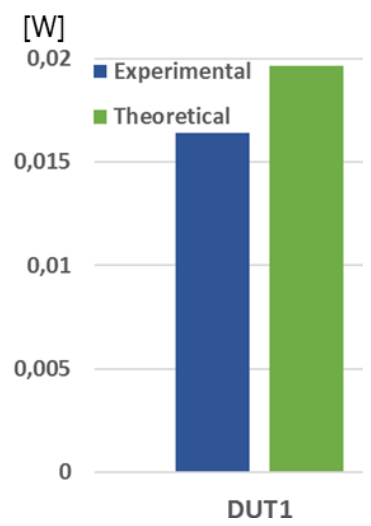

(c)

Figure 5 (a)Measured vs. Theoretical conduction losses $\left(\mathrm{P}_{\mathrm{ON}}\right)$ at $10 \%$ load, (b) driving power losses $\left(\mathrm{P}_{\mathrm{DR}}\right)$ breakdown for DUT1 at $10 \%$ load and (c) experimental vs. theoretical driving-on losses at $10 \%$ load

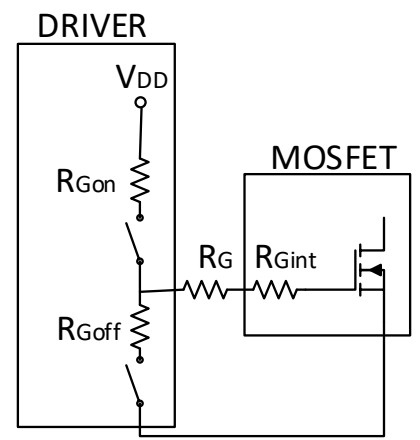

Figure 6 Driver and MOSFET gate resistors 


\section{NEW ELECTRICAL CHARACTERIZATION}

As has been shown, $\mathrm{P}_{\mathrm{SW}}$ losses are significant, especially at low and medium load, even if the converter is working with ZVS. It is important to predict and quantify those additional soft-switching losses, Esw_SOFT [8].

The quantification of $\mathrm{E}_{\text {SW_SOFT }}$ for SJ-MOSFETs in LLC resonant converters has drastically changed during the last decade. Despite some initial works neglecting them [5], other investigations point out to an increasing $\mathrm{E}_{\mathrm{SW}}$ _SOFT predominance in modern soft-switching converters [9]. Efforts have been done to include non-linear capacitance effects [10]-[11] and non-ZVS operation [12] in MOSFETs simulation models. However, the most recent discoveries in output capacitance $\left(\mathrm{C}_{\mathrm{OSS}}\right)$ hysteresis for SJ-MOSFETs are not considered in those models [13]-[16].

A physical relationship between unexpected ZVS power loss and $\mathrm{C}_{\mathrm{OSS}}$ hysteresis was introduced in [17] for SJ MOSFETs. The experimental observations published in [13] were qualitatively reproduced in [17], elucidating the existence of $\mathrm{E}_{\mathrm{SW} \_ \text {_SOFT }}$ during $\mathrm{C}_{\mathrm{OSS}}$ charge and discharge.

The value of $\mathrm{E}_{\mathrm{SW} \_ \text {sofT }}$ varies from device to device in function of geometrical and technological features. Furthermore, no information on this effect is provided in datasheets, application notes or simulation models. In fact, Coss provided by device vendors is typically extracted by small-signal techniques when only large-signal analysis captures Coss hysteresis.

In order to obtain a new explicative FoM, an Auriga pulsed I-V system [18] is proposed for $E_{S W \_s O F T}$ characterization. Differently from other reported techniques [19]-[21], this is the first suggested commercial system. The characterization system that this hardware delivers is able to capture measurements with very high speed and resolution (up to $0.01 \%$ of $\max$ current), and it is temperature independent. Moreover, voltage/current measurements have emerged as the preferred method of getting different characteristics of active devices.

Figure 7a shows an example of some measurements done to extract $\mathrm{E}_{\mathrm{SW} \text { _SOFT }}$ (by applying a $400 \mathrm{~V}$ pulse on the device with the period/frequency desired, and measuring $\mathrm{I}_{\mathrm{D}}, \mathrm{V}_{\mathrm{DS}}$ and the cumulative energy $-\mathrm{E}_{\mathrm{ACC}}-$ waveforms). Concretely, ESW_SOFT is considered as the energy accumulated after applying a complete cycle of discharge-charge to the device.

Based on the information extracted, the mechanism to detect $\mathrm{C}_{\text {oss }}$-hysteresis can be seen in Figure $7 \mathrm{~b}$ (by dividing the current by the derivative of the voltage), noticeable and measurable during the charge and discharge of the SJMOSFETs. This effect is an inherent loss mechanism for SJMOSFETs due to their inner structure. Differently from the information usually provided by the manufacturer (Figure 7, small signal waveform, in red), this characterization shows dissimilarities between the charge and discharge of the devices (large signal, in blue) which states and validates an intrinsic energy loss.

By using this characterization method, Figure $7 \mathrm{c}$ compares the new FoM defined as $\mathrm{R}_{\mathrm{ON}} * \mathrm{E}_{\mathrm{SW} \_ \text {SOFT }}$ for different technologies (GaN E-Mode devices, SiC MOSFETs and some of the DUTs characterized in this paper). This FoM which considers both $\mathrm{R}_{\mathrm{ON}}$ (important for heavy loads) and ESW_SOFT (crucial for low and medium loads) qualitatively matches with efficiencies (Figure $3 \mathrm{a}$ ) and $\mathrm{P}_{\mathrm{sw}}$ trends ( Figure
4), since the experimental results show that $\mathrm{DUT}_{1}$ presents better performance than $\mathrm{DUT}_{5}$ or $\mathrm{DUT}_{4}$, and it can be extended to the rest of devices under test.

It is worth to remark that other technologies ( $\mathrm{GaN}, \mathrm{SiC})$ for the same range of voltage promise better performance according to the proposed FoM.

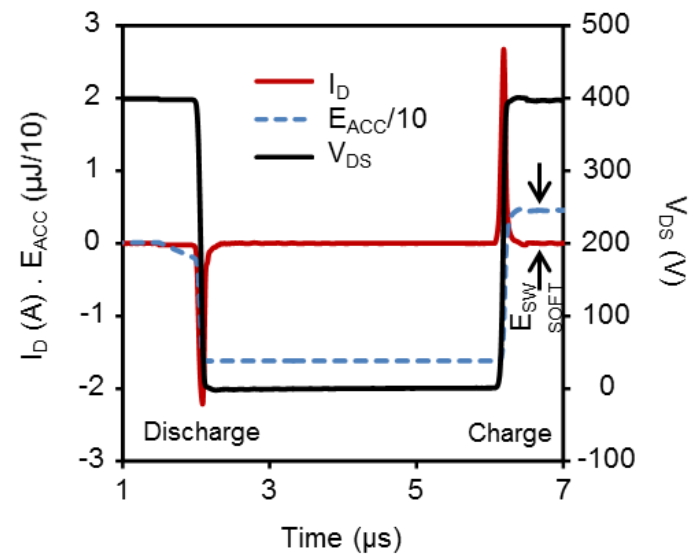

(a)

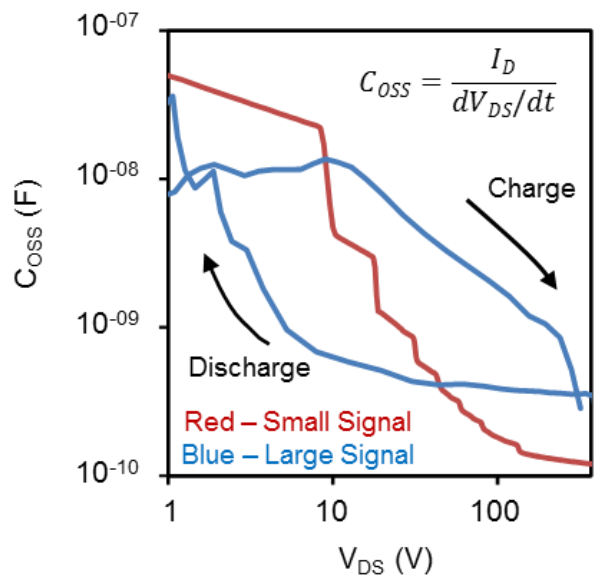

(b)

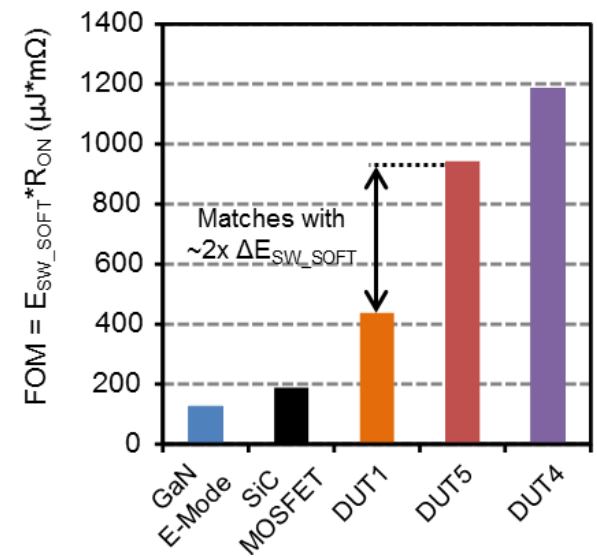

(c)

Figure 7 Auriga pulsed I-V tests: (a) $\mathrm{I}_{\mathrm{D}}, \mathrm{V}_{\mathrm{DS}}$ and $\mathrm{E}_{\mathrm{ACC}}$ waveforms, (b) $\mathrm{C}_{\mathrm{OSS}}$ hysteresis, (c) $\mathrm{R}_{\mathrm{ON}} * \mathrm{E}_{\mathrm{SW}-\mathrm{SOFT}}$ in SJ-MOSFETs, GaN E-Mode and $\mathrm{SiC}$ MOSFET 


\section{CONCLUSIONS}

This paper presents an original perspective of the requirements and trends in power transistors for LLC primary side converters by covering new insights in power loss analysis and application-oriented characterization techniques.

A deep power loss model, which takes into account PON, PDR and PSW contributions, is followed and compared with experimental results, showing a good match.

Up to now, existing FoMs are not enough to select the optimum power transistors for LLC primary side. New FoMs which consider $\mathrm{P}_{\mathrm{DR}}$ and $\mathrm{P}_{\mathrm{SW}}$, especially relevant at low and medium loads, are needed. Eventually, a new I-V based E $_{\text {SW_SOFT }}$ characterization method is proposed and explained and a new FoM based on this intrinsic energy is described.

\section{ACKNOWLEDGMENT}

This work has been supported by the Spanish Government under Project MINECO-17-DPI2016-75760-R, Project DPI2014-56358-JIN and grant FPI BES-2014-070785, and by the Principality of Asturias under Project SV-PA-17-RIS3-4 and FEDER funds.

\section{REFERENCES}

[1] V. Vorperian And Slobodan Cuk. "A Complete DC Analysis of The Series Resonant Converter." IEEE Power Electronics Specialists conference (1982): 85 - 100

[2] Deng, Junjun, Siqi Li, Sideng Hu, Chunting Chris Mi, and Ruiqing Ma "Design methodology of LLC resonant converters for electric vehicle battery chargers." IEEE Transactions on Vehicular Technology 63, no. 4 (2014): 1581-1592.

[3] H. Hu, X. Fang, F. Chen, Z. J. Shen, and I. Batarseh, "A modified high-efficiency LLC converter with two transformers for wide input-voltage range applications," IEEE Trans. Power Electron., vol. 28, no. 4, pp. 1946-1960, Apr. 2013

[4] Webpage: http://www.80PLUS.org [last access: June 2018]

[5] Person, C. E. "Selection of primary side devices for LLC resonant converters". Doctoral dissertation, Virginia Tech, 2008

[6] A. Steiner, F. Di Domenico, J. Catly, F. Stückler, "600W half bridge LLC Evaluation Board with 600V CoolMOSTM C7", Infineon Technology AN - June 2015
[7] "Eice DRIVER Compact. 2EDL family. Technical Description". Infineon Technology AN2013-11. April 2015

[8] J. Roig et al., "High-accuracy modelling of ZVS energy loss in advanced power transistors," 2018 IEEE Applied Power Electronics Conference and Exposition (APEC), San Antonio, TX, 2018, pp. 263269.

[9] K. Tabira, S. Watanabe, T. Shimatou, and T. Watashima, "Advantage of super junction MOSFET for power supply application," in 2014 IEEE Power Electronics Conference (ECCE-ASIA), pp. 2939-2943, 2014.

[10] R. Elferich, "General ZVS half bridge model regarding nonlinear capacitances and application to LLC design," in 2012 IEEE Energy Conversion Congress and Exposition (ECCE), pp. 4404-4410, 2012.

[11] D. Costinett, D. Maksimovic, and R. Zane, "Circuit-oriented treatment of nonlinear capacitances in switched-mode power supplies," IEEE Transactions on Power Electronics, 30(2), pp. 985-995, 2015.

[12] C. Oeder, M. Barwig, and T. Duerbaum, "Estimation of switching losses in resonant converters based on datasheet information," in IEEE European Conference on Power Electronics and Applications (EPEECCE Europe), pp. 1-9, 2016.

[13] J. B. Fedison, M. Fornage, M. J. Harrison, and D. R. Zimmanck, "Coss related energy loss in power MOSFETs used in zero-voltage-switched applications," in 2014 IEEE Applied Power Electronics Conference and Exposition (APEC), pp. 150-156, 2014.

[14] R. Miftakhutdinov, "New ZVS analysis of PWM converters applied to super-junction, GaN and SiC power FETs," in 2015 IEEE Applied Power Electronics Conference and Exposition (APEC), pp. 336-341, 2015.

[15] X. Li and A. Bhalla, "Comparison of intrinsic energy losses in unipolar power switches," in 2016 IEEE Wide Bandgap Power Devices and Applications (WiPDA), pp. 228-232, 2016.

[16] M. Kasper, R. M. Burkart, G. Deboy, and J. W. Kolar, "ZVS of power MOSFETs revisited," IEEE Transactions on Power Electronics, 31(12), pp. 8063-8067, 2016.

[17] J. Roig and F. Bauwens, "Origin of Anomalous Coss Hysteresis in Resonant Converters With Superjunction FETs," IEEE Transactions on Electron Devices, 62(9), pp. 3092-3094, 2015

[18] Webpage: https://focus-microwaves.com/pulsed-iv/

[19] Kasper M. et al. "ZVS of power MOSFETs revisited". IEEE Transactions on Power Electronics, 31(12), 8063-8067, 2016.

[20] Fedison, J. B., and M. J. Harrison. "COSS hysteresis in advanced superjunction MOSFETs“. In APEC'16, pp. 247-252, 2016.

[21] R. Miftakhutdinov. "Analysis and Practical Method of Determining WBG FET Switching Losses Associated with Nonlinear Coss“. APEC'16, 2016. 